\title{
A Finite Capacity of Production Planning Approach for Industrial Manufacturing
}

\author{
Aboubaker Altiaieb Moussttfa ${ }^{1 *}$, Dušan Malinžák ${ }^{2}$ \\ Technical university of Košice, Faculty of Mechanical Engineering, Department of Industrial Engineering and Management
}

\section{KEY WORDS}

Capacity planning, production models, planning, MTO, S\&OP.

\begin{abstract}
This paper discusses a production planning approach that takes the finite capacity of the manufacturing system into account. The approach is based on a reduced level of detail modeling of the process flows. The paper considers explicitly only bottleneck steps. A mixed integer programming (MIP) formulation is suggested to determine completion time targets for the bottleneck steps of the lots. A Lagrange relaxation technique is applied to solve the MIP approximately. Intermediate capacity management is related to rough-cut capacity planning, linked to the master schedule, and capacity requirements planning, and linked to the material requirements plan.
\end{abstract}

\section{Introduction}

Therefore, the development of efficient planning and control strategies is very beneficial in the industry manufacturing domain. This paper focuses on a production planning problem in industry wafer fabrication facilities. We are interested in determining completion time targets for processing steps on bottleneck machines. These time targets can be used by a corresponding dispatch approach or within hierarchical production planning and scheduling approaches [1].

Manufacturing firms apply various policies for fulfilling customer orders. Some firms choose to fill orders through finished goods inventory. Such a policy is referred to in the literature as make-to-stock (MTS). Other firms choose to start working on an order only after it has been placed. Such a policy is referred to as make-to-order (MTO). There are a variety of MTO operations, depending on the timing the manufacturer gets involved in the product's life cycle [1].

The major difference between MTO and MTS is that MTS makes standard products using a standardized process, which do not exist for MTO at the time of capacity planning. Unlike in MTS, which hold finished goods in inventory as a buffer against variations in customer demand, MTO operations hold capacity in reserve to meet customer demand. The most important aspect in MTO is the effective and efficient use of available capacity to meet customer demands. Since unused capacity represents a loss in revenue, an MTO operation manager needs to be conservative for holding their capacity.

\section{Production and Capacity Planning}

The goal of production planning is simply to maintain flow, whereas the goal of 
capacity planning is to maintain a flow in resource usage. Much in the way that a person adjusts faucet spigots to achieve a desired temperature, the individual in charge of this type of planning adjusts the workforce and process flow to obtain a regular use of company resources with minimal downtime, minimal bottlenecks and some level of output consistent with all the resources being put in the process. [1], [2].

\subsection{Production planning}

Production planning, or production scheduling, is a term assigned to the planning of production in all aspects, from workforce activities to product delivery. Production planning is almost exclusively seen in manufacturing environments; however, many of the techniques employed in production planning can be and are used by many serviceoriented businesses. Understanding the behavior of a process, finding bottlenecks, reducing workin-process inventories, developing optimal scheduling, forming optimal forecasting methods, and polishing inventory control methods are the main concerns of production planning.

In a nutshell, production planning is primarily concerned with the efficient use of resources. While it is sometimes referred to as operations planning, and truly employs many of the same techniques, the primary distinguishing characteristic is that production planning is focused on the actual production whereas operations planning looks at the operation as a whole [2], [3], [4].

\subsubsection{Aspects of production planning}

Production is planned with either a long-term, medium-term or short-term view. Long-term views focus on the major decisions a company makes that influence capacity whereas short-term views focus more on using what a company currently has more efficiently. Medium-term views focus more on adjustments, such as hiring, firing, layoffs, increasing inventory or expecting back orders.

\subsection{Capacity planning}

Capacity can be a difficult concept to quantify. Maximum output is calculated by identifying the maximum output of a given period when demand had been highest and assuming that that level of performance could be reproduced on a daily basis; however, this is generally not sustainable and can lead to problems fulfilling demand. Instead, capacity planning is focused on maximizing the capacity of a company in a way that allows it to be more efficient and, thusly, more profitable. Capacity planning at its most basic attempts to match the volume the company is able to produce to the demand in order to avoid downtime by preventing bottlenecks. [3], [1].

Too much capacity can result in a low return on asset investment, whereas as too little capacity can drive away customers. A good capacity plan has a level amount of input (raw materials and other resources) for its output (the actual product) with little to no bottlenecks and little to no downtime.

\subsubsection{Methods of capacity planning}

One popular method of capacity planning is aggregate planning. Aggregate planning basically ties facility planning in with scheduling decisions, and it does so in a way that is quantitative, meaning it produces numbers to back up an operations plan. Plans generally either "chase" demand, adjusting its workforce accordingly or are "level" plans, meaning that labour is relatively constant with fluctuations in demand being met by inventories and back orders. Plans may also be "hybrid," meaning that they combine these two approaches.

Another popular method of capacity planning is the use of the Theory of Constraints (TOC). TOC serves to answer the question of what to change by using cause-and-effect modelling. It operates on the basic premise that a system can never be better than the weakest part thereof and that solving the problem of what holds the system back depends upon the identification of that constraint and the mitigation thereof. This process is frequently likened to that of a physician diagnosing a patient, designing a treatment plan and executing that plan. TOC is a useful tool in project management because of its ability to look at a system specifically and postulate as to how strong it could be. This methodology is quite useful in creating a starting point toward a solution to various business problems from marketing to supplier relationships to project management [2], [3], [4], [5].

\section{Analytical Models}

Production planning problems are one of the most interesting applications for optimization tools using mathematical programming. The idea of incorporating uncertainty in mathematical models appears initially with Dantzig, (Dantzig, 1955). 


\subsection{Hierarchical production planning}

One of the important advances in the field of production planning by means of mathematical programming was the concept of hierarchical production planning (Hax and Meal, 1975). Hierarchical production planning has attracted wide research activity, including the addition of parameters with uncertainty. Gfrerer and Zapfel (1995) present a multi-period hierarchical production planning model with two planning levels, i.e. aggregate and detailed, and with uncertain demand. Meybodi and Foote (1995), on the other hand, develop a multi-period model for hierarchical production planning and scheduling with random demand and production failure. Zapfel (1996) presents a hierarchical model that can be incorporated in a MRP II system to program the production with demand uncertainty.

\subsection{Capacity planning}

Bitran and Yanesse (1984) propose deterministic approximations to a non-sequential capacity planning model and analyse its effectiveness when the demand is characterized by standard probability distributions. Eppen et al. (1989) model the strategic capacity planning problem of a major automobile manufacturer. They use a stochastic programming approach based on demand scenarios with emphasis on longer-range decisions regarding facility selection for manufacturing. Paraskevopoulos et al. (1991) describe a production capacity planning problem with uncertain demand. They use a robust approach avoiding the inherent complexities in a nonlinear stochastic formulation. Karabuk and Wu (1999) develop a strategic capacity planning model for a major semi-conductor manufacturer. They formulate a multi-stage stochastic program with recourses, where demand.

\subsection{Manufacturing resource planning}

An integrated information system is used by businesses. Manufacturing Resource Planning (MRP II) evolved from early Materials Requirement Planning (MRP) systems by including the integration of additional data, such as employee and financial needs. The system is designed to centralize, integrate and process information for effective decision making in scheduling, design engineering, inventory management and cost control in manufacturing. Manufacturing resource planning, also known as MRP II, is a method for the effective planning of a manufacturer's resources. MRP II is composed of several linked functions, such as business planning, sales and operations planning, capacity requirements planning, and all related support systems. The output from these MRP II functions can be integrated into financial reports, such as the business plan, purchase commitment report, shipping budget, and inventory projections. It has the capability of specifically addressing operational planning and financial planning, and has simulation capability that allows its users to conduct sensitivity analyses (answering "what if" questions) [5], [6], [7], [8].

The earliest form of manufacturing resource planning was known as material requirements planning (MRP). This system was vastly improved upon until it no longer resembled the original version. The newer version was so fundamentally different from MRP, that a new term seemed appropriate. Oliver Wight coined the acronym MRP $\|$ for manufacturing resource planning [5], [6], [7], [8]. In order to best understand MRP II, one must have a basic understanding of MRP, so we will begin with a look at MRP and then expand into MRP II.

\subsubsection{Material requirements planning}

Material requirements planning (MRP) is a computer-based, time-phased system for planning and controlling the production and inventory function of a firm from the purchase of materials to the shipment of finished goods. All MRP systems are computer based since the detail involved and the inherent burden of computation make manual use prohibitive. MRP is time phased because it not only determines what and how much needs to be made or purchased, but also when. Material requirements planning first appeared in the early 1970s and was popularized by a book of the same name by Joseph Orlicky. Its use was quickly heralded as the new manufacturing panacea, but enthusiasm slowed somewhat when firms began to realize the difficulty inherent in its implementation [7], [8].

The MRP system is composed of three primary modules, all of which function as a form of input. These are the master production schedule, the bill-of-materials, and the inventory status file. Each module serves a unique purpose that is inter-related with the purpose of the other modules, and produces several forms of usable output [7], [8]. 


\subsubsection{Master production schedule}

The master production schedule (MPS) is basically the production schedule for finished goods. This schedule is usually derived from current orders, plus any forecast requirements. The MPS is divided into units of time called "buckets." While any time frame may be utilized, usually days or weeks is appropriate. The MPS is also said to be the aggregate plan "disaggregated." In other words, the plan for goods to be produced in aggregate is broken down into its individual units or finished goods [7], [8].

\section{The Capacity Planning Problem in Make-to- Order Enterprises- Short Term Capacity Planning}

The short-term capacity planning sets a daily or weekly capacity plan for a planning horizon, long enough to accommodate each order's lead time. The objective of short-term capacity planning is to ensure an appropriate match between the resources availability and the capacity requirement for a production plan at the work center level [9]. For an MTO operation, it has to specify resources requirement of each labor and machine type for each customer order at its component level. Each customer order first is translated into internal orders and detailed work orders, which are then summarized into a load schedule (time-phased capacity requirements) by labor and/or equipment, in coordination with materials arrival. A typical MTO operation routinely considers the use of alternative sources such as overtime and outsourcing, in order to meet work order's deadline. To assure a smooth production, an MTS operation usually imposes a freeze period, in which no change to the production plan can be made. In an MTO operation, however there is no freeze period imposed [9], [10], [11].

The model helps to unify two decisions: which orders to accept and how much capacity is required of each resource in each source in order to complete an accepted order. The secondary research objective is to demonstrate the usefulness of the model by illustrating examples where conventional wisdom is not always profitable. The conventional wisdom is to maximize the utilization of the resources, but in MTO environment this does not guarantee profit maximization. The inputs to the model are the potential customer orders and their characteristics (such as process route, processing times, selling price, etc.) and job shop configuration (such as resources, their availability, etc.). The output from the model would be the list of accepted orders and their schedules.

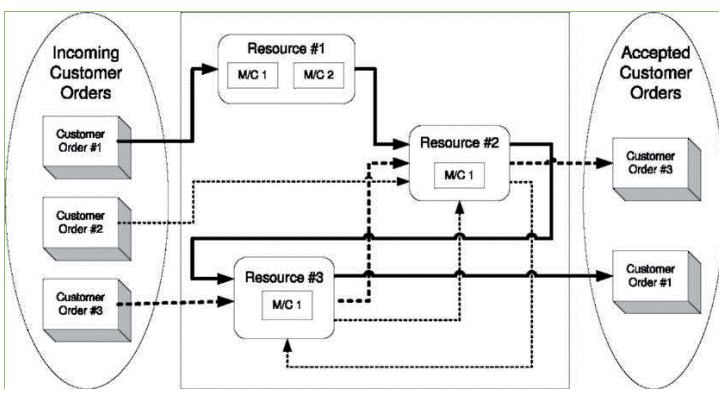

Fig. 1: (a) Graphical illustration of the problem under study.

\section{Conclusion}

In this paper, two different approaches to capacity planning problem mainly considered on time management are mentioned. I would like to stress, that there is no better approach, long- term capacity planning, or short-term capacity planning. Both are important and forgetting one of them can cause serious damages.

\section{References}

[1] Hayes, R.,H., Wheelwright, S.,C.. 1984. Restoring our Competitive Edge - Competing Through Manufacturing. Wiley. New York

[2] Leong, K., Snyder, D.,L., Ward, P., T.. 1990. Research in the process and content of manufacturing strategy. Omega.

[3] O'Farrell, R.. 1999-2013. eHow Contributor. Ehow Money, Production \& Capacity Planning. Demand Media.

[4] Ling, R., C., Goddard, W., E.. 1988. Orchestrating Success Improve Control of the Business with Sales and Operations Planning. Wiley. New York.

[5] Stahl, R., A.. 1995. Sales and operations planning (S\&OP) Fundamental that still works, Proceedings of APICS. 38th International Conference. Orlando.

[6] Olhager, J., Rudberg, M., Wikner, J.. 2001. Long-term capacity management: Linking the perspectives from manufacturing strategy and sales and operations planning. Int. J. Production Economics 69.

[7] Cochran, J., K., Uribe, A., M.. 2005. A Set Covering Formulation for Agile Capacity Planning Within Supply Chains. International Journal of Production Economics 95.

[8] Jonsson, P., Stig-Arne M.. 2002. Use and Applicability of Capacity Planning Methods. Production and Inventory Management Journal 43.

[9] Schonberger, R., J., Knod, E., M., JR.. 1991. Operation man- 
agement: Improving customer services. Boston.

[10] Hayes, R.,H., Wheelwright, S.,C.. 1984. Restoring our competitive edge: Competing through manufacturing. Wiley. New York.

[11] Chen, Ch., Mestry,S., Damodaran.P., Wang, Ch.. 2009. The capacity planning problem in make-to-order enterprises, Mathematical and Computer Modelling journal homepage. Elsevier. 